\title{
Cholesterol Efflux Potential of Sera from Mice Expressing Human Cholesteryl Ester Transfer Protein and/or Human Apolipoprotein Al
}

\author{
V. Atger, ${ }^{\star}$ M. de la Llera Moya, ${ }^{\ddagger}$ M. Bamberger, ${ }^{\S}$ O. Francone, ${ }^{\$}$ P. Cosgrove, ${ }^{\S}$ A. Tall," A. Walsh, ${ }^{,}$N. Moatti, ${ }^{\star}$ and \\ G. Rothblat ${ }^{\ddagger}$ \\ ${ }^{*}$ Hospital Broussais, Paris, France; ${ }^{\ddagger}$ Medical College of Pennsylvania and Hahnemann University, Philadelphia, Pennsylvania 19129; \\ ${ }^{\S}$ Pfizer Central Research, Groton, Connecticut 06340; "Columbia University, New York 10027; and 'The Rockefeller University, New \\ York 10021
}

\begin{abstract}
The ability of whole serum to promote cell cholesterol efflux and the relationships between apoprotein and lipoprotein components of human serum and efflux have been investigated previously ( de la Llera Moya, M., V. Atger, J. L. Paul, N. Fournier, N. Moatti, P. Giral, K. E. Friday, and G. H. Rothblat. 1994. Arterioscler. Thromb. 14:1056-1065). We have now used this experimental system to study the selective effects of two human lipoprotein-related proteins, apoprotein AI (apo AI) and cholesteryl ester transfer protein (CETP) on cell cholesterol efflux, when these proteins are expressed in transgenic mice. The percent efflux values for cholesterol released in $4 \mathrm{~h}$ from Fu5AH donor cells to $5 \%$ sera from the different groups of mice were in the order: background = human apo AI transgenic (HuAITg) $>$ human CETP transgenic (HuCETPTg) $>$ human apo AI and CETP transgenic (HuAICETPTg) $\gg$ apo AI knockout mice. In each group of mice a strong, positive correlation ( $r^{2}$ ranging from 0.64 to 0.76 ) was found between efflux and HDL cholesterol concentrations. The slopes of these regression lines differed between groups of mice, indicating that the cholesterol acceptor efficiencies of the sera differed among groups. These differences in relative efficiencies can explain why cholesterol efflux was not proportional to the different HDL levels in the various groups of mice. We can conclude that: ( $a$ ) HDL particles from HuAITg mice are less efficient as cholesterol acceptors than HDL from the background mice; (b) despite a lower average efflux due to lower HDL cholesterol concentrations, HDL particles are more efficient in the HuCETPTg mice than in the background mice; and (c) the coexpression of both human apo AI and CETP improves the efficiency of HDL particles in the HuAICETPTg mice when compared with the HuAITg mice. We also demonstrated that the esterification of the free cholesterol released from the cells by lecithin cholesterol acyltransferase in the serum was reduced in the HuAITg and AI knockout mice, whereas it was not different from background values in the two groups of mice expressing human CETP. (J. Clin.
\end{abstract}

Address correspondence to G. Rothblat, Department of Biochemistry, Medical College of Pennsylvania, 2900 Queen Lane, Philadelphia, PA 19129. Phone: 215-991-8308; FAX: 215-843-8849.

Received for publication 24 February 1995 and accepted in revised form 24 August 1995.

J. Clin. Invest.

(C) The American Society for Clinical Investigation, Inc. $0021-9738 / 95 / 12 / 2613 / 10 \$ 2.00$

Volume 96, December 1995, 2613-2622
Invest. 1995. 96:2613-2622.) Key words: transgenic mice • lipoprotein • tissue culture cells $\cdot$ lipids $\cdot$ sterols

\section{Introduction}

Elevated concentrations of high density lipoproteins (HDL) have been correlated with a reduced risk of coronary artery disease (1), and this protective effect of HDL is thought to be linked to the proposed role of HDL in the process termed reverse cholesterol transport (RCT). ${ }^{1}$ In this process HDL, or specific subclasses of HDL, removes excess cholesterol from peripheral cells, and through a series of related pathways this cholesterol is returned to the liver for excretion or conversion to bile acids (2). It is proposed that the reverse cholesterol pathway is responsible for the maintenance of cholesterol levels in peripheral tissues and for the elimination of excess cholesterol from foam cells (3). The first step in this RCT pathway is the movement of cholesterol out of cells onto the lipoprotein acceptors in the interstitial fluid, and although a wide variety of native and reconstituted acceptor particles has been shown to stimulate cell cholesterol efflux, the contribution of the specific lipoprotein fractions of serum responsible for this process remains to be established. In addition, serum factors such as lecithin cholesterol acyl transferase (LCAT) (2) and cholesteryl ester transfer protein (CETP) (4) play an important role in the reverse cholesterol transport process.

Identification of the components in serum, and a quantitation of their contribution to efflux and RCT, is best achieved through the use of whole serum as the cholesterol acceptor, since all of the apoproteins, lipoproteins, transfer factors, and enzymes that participate in RCT are present. Although there is an extensive literature on the efflux of cell cholesterol to isolated populations of acceptor particles, studies using whole serum to stimulate efflux are limited. Two general approaches have been used for whole serum efflux studies: experiments in which the donor cells are incubated for short periods (1-5 $\mathrm{min}$ ) in the presence of undiluted $(100 \%)$ plasma, and experiments using diluted serum and incubation times ranging from a few minutes to many hours. The former type of study is best represented by the investigations of Fielding and colleagues (5-7). They have demonstrated that a particularly efficient acceptor in the initial removal of cholesterol from cells is a small, phospholipid-rich, apo AI-containing lipoprotein $(8-10)$ that has been identified

1. Abbreviations used in this paper: CETP, cholesteryl ester transfer protein; GGE, gradient gel electrophoresis; HuAICETPTg, transgenic mice expressing human apo AI and CETP; HuAITg, transgenic mice expressing human apo AI; HuCETPTg, transgenic mice expressing human CETP; KO, knockout; LCAT, lecithin cholesterol acyl transferase; RCT, reverse cholesterol transport; REP, relative efflux potential. 
in both plasma and lymph, and has been characterized as a pre $\beta$-migrating HDL $(7,10-12)$. As another approach, we have recently developed an experimental system in which the donor cells are exposed to diluted serum for periods ranging from 30 min to a number of hours (13). This system is particularly suitable for the screening of large numbers of serum samples for their ability to stimulate cholesterol efflux (i.e., efflux potential). Measurement of the efflux potential of more than 100 specimens of human serum established a series of correlations between efflux and serum components. However, the complex mixture of proteins and the heterogeneity of the lipoproteins in human serum complicates the assessment of the specific roles played by individual proteins or lipoproteins in the early steps of RCT.

The recent development of a large variety of transgenic strains of mice (14-17) has provided us the opportunity to determine how the expression of specific serum factors influences the ability of serum to promote the efflux of cellular cholesterol. The two factors that were investigated in the present study were apo AI and CETP, two proteins that play a major role in HDL structure and metabolism. The role of CETP in remodeling HDL particle distribution is well documented (4, 18 ) and the influence of human apo AI on HDL particle size and distribution has been clearly demonstrated in studies of transgenic mice conducted in a number of laboratories $(18,19)$. In this investigation, we have measured the efflux potential of serum obtained from transgenic mice expressing either human apo AI $(19,20)$, human CETP (21), or both apo AI and CETP $(14,17)$. We have also determined the efflux potential of serum obtained from mice in which the mouse apo AI gene has been inactivated (apo AI KO). In addition, the experimental system that we used allowed the measurement of the fractional esterification of the cholesterol released from the cells (LCAT), and quantitated the effect of human apo AI and CETP expression on the relative LCAT activity in the mouse sera.

\section{Methods}

Animal strains, chemical analysis, and materials. Transgenic mice expressing human apo AI, line 427 originally described by Walsh et al. (19), were obtained from Charles River Laboratories (Wilmington, MA). Males homozygous for the transgene were bred against C57B1/6 females. Offspring positive for the transgene were used. Mice expressing human apo AI and human CETP, originally described by Hayek et al. (14), were bred with each other to obtain animals expressing CETP alone or both CETP and apo AI. The apo AI knockout mice (AI KO) were as described by Plump et al. (22). At time of killing, serum from these animals was collected and frozen using a standardized method (13). Total serum and HDL cholesterol in samples were assayed using standard phosphotungstic acid precipitation and enzymatic methods (23). Human apo AI was measured by a sandwich ELISA. Assay plates (Maxisorb; Nunc, Roskilde, Denmark) were incubated overnight with monoclonal antibodies against human apo $\mathrm{AI}$ obtained from Chemicon International, Inc. (Temecula, CA). Plates were blocked for $1 \mathrm{~h}$ with PBS containing $1 \%$ BSA (type V). Serum from transgenic mice was diluted $1: 10^{6}$ in blocking buffer containing $0.004 \%$ Tween 20 and a 1:1,000 dilution of polyclonal goat antisera against human apo AI (Chemicon) and incubated for $3 \mathrm{~h}$. Subsequently, a rabbit anti-goat IgG conjugated to alkaline phosphatase (Sigma Immunochemicals, St. Louis, MO) was used to quantitate the bound human apo AI using a standard colorimetric assay (Sigma). Purified apo AI (Sigma) was used to generate the standard curve. CETP levels were measured by a monoclonal-based immunoassay (24). Specimens from AI KO mice were provided by The Rockefeller University and were analyzed for serum total cholesterol and HDL cholesterol, as described previously (19). Human HDL was isolated from serum by density centrifugation and apo AI was prepared from delipidized human HDL as described previously (25).

Efflux assay. The efflux potential of serum samples from individual mice was assayed as described previously (13). Briefly, diluted serum samples from individual mice were incubated with $\left[{ }^{3} \mathrm{H}\right]$ cholesterollabeled Fu5AH rat hepatoma cells for various lengths of time $(0.5-4$ h) at $37^{\circ} \mathrm{C}$. The radioactivity released to the medium was determined as the fraction of the total lipid-soluble label per well of cells to obtain fractional efflux value. Most efflux studies were done with a $5 \%$ serum dilution; in a few experiments efflux assays were done in parallel with 5 and $2.5 \%$ serum and efflux was proportional to the dilution used. Aliquots from pools of human and mouse sera were obtained from commercially available (Taconic Farms, Germantown, NY) outbred Swiss White [ Tac- $(\mathrm{SW})-\mathrm{fBr}$ ] and $\mathrm{B} 6 \mathrm{C} 3 \mathrm{~F} 1$ mice, referred to as control serum, and were assayed in every experiment to monitor interassay variability. Other normal mouse serum came from Pfizer Central Research and included serum from C57BL $\times$ CBA hybrids, designated in this study as background serum. All efflux values are averages of three determinations. Maximal efflux values $\left(V_{\max }\right)$ were derived from the analysis of the results of dose-dependent curves plotted as $\mathrm{V}$ ( $\%$ efflux/ h) vs. $\mathrm{V} /[\mathrm{S}]$ (\% serum), in analogy with a Hofstee plot used to determine maximal velocities for enzyme reactions (26). In this plot, the $y$ axis intercept is the estimated maximum rate of cholesterol release at infinite acceptor concentration, and the serum concentration at which half of the maximal rate for efflux is reached, the $\mathrm{EC}_{50}$, is determined by the slope of the regression line.

In some experiments, mouse $\mathrm{L}$ cell fibroblasts were used as cholesterol donor cells. Mouse L cells were grown in 24-well plates using MEM containing $10 \%$ FBS. L cells were radiolabeled $(2 \mu \mathrm{Ci} /$ well $)$ and enriched with cholesterol by incubating with MEM containing $200 \mu \mathrm{g} /$ $\mathrm{ml}$ cholesterol added as a $2: 1$, cholesterol/phosphatidylcholine dispersion as described previously (27).

Assay for esterification of released cellular cholesterol. After exposure to the cholesterol donor cells, the efflux medium containing the test sera was collected and cooled to $0^{\circ} \mathrm{C}$ in an ice bath. An aliquot of this medium was extracted using the method of Bligh and Dyer (28) and the radiolabeled cholesterol and cholesteryl ester present were separated using TLC and quantified as described (13). The amount of radiolabeled cholesterol esterified extracellularly was expressed as percent esterification $/ 4 \mathrm{~h}$ and this value was taken to be an indication of the LCAT activity of the test sera (13). Pools of both human and normal mice sera were used to monitor interassay variability.

Characterization of serum HDL particles. HDL particles were separated by size using native gradient gel electrophoresis (GGE). Precast polyacrylamide gels (4-15\%) were obtained from BioRad Laboratories (Anaheim, CA) and were used for electrophoresis at $4^{\circ} \mathrm{C}$ in Tris-borateEDTA running buffer at $\mathrm{pH}$ 7.4. The lipoproteins in the mouse serum specimens were stained with Sudan black B before electrophoresis as described previously (29). Briefly, 3 vol of serum were mixed with 4 vol of a staining and loading solution and incubated for 15-30 min at room temperature. The staining and loading solution contained $2 \mathrm{vol}$ of a 1:50 dilution in ethylene glycol of Sudan black B and $1 \mathrm{vol}$ of glycerol. Gels were prerun at $50 \mathrm{~V}$ for $30 \mathrm{~min}$ and for $2 \mathrm{~h}$ at $200 \mathrm{~V}$ once the samples were loaded. Western blots of the gels were prepared using a standard procedure (30). Lipoprotein fractions were immunostained using a polyclonal rabbit anti-mouse apo AI antibody.

Two-dimensional electrophoresis was carried out as described previously by Francone et al. (31 ). Briefly, $15 \mu \mathrm{l}$ of serum was electrophoresed in $0.75 \%$ (wt/vol) agarose gel in $50 \mathrm{mM}$ barbital buffer, at $\mathrm{pH}$ 8.6, on Gelbond (FMC Corp. Bio Products, Rockland, ME). Electrophoresis in the second dimension was carried out on a $2-16 \%$ polyacrylamide gradient gel (Integrated Separation Systems, Natick, MA) and transferred to NitroPlus membranes (Micron Separations, Westborough, MA). To identify the human apo AI-containing HDL species, nitrocellulose membranes were incubated for $2 \mathrm{~h}$ at room temperature with $2 \%$ milk in $10 \mathrm{mM}$ phosphate buffer, at $\mathrm{pH} 7.4$, and then with a biotinylated 
Table I. Serum Parameters of Control and Transgenic Mice

\begin{tabular}{lcccccc}
\hline \multicolumn{1}{c}{ Mouse } & Tot. chol. & $n$ & HDL-C & $n$ & apo AI* & CETP \\
\hline & $m g / d l \pm S D$ & & $m g / d l \pm S D$ & & & $m g / d l \pm S D$ \\
Background & $103 \pm 15$ & 17 & $74 \pm 13$ & 14 & & \\
HuAITg & $142 \pm 25$ & 29 & $114 \pm 25$ & 16 & $58 \pm 200$ & 17 \\
HuCETPTg & $81 \pm 12$ & 17 & $45 \pm 9$ & 10 & & \\
HuAICETPTg & $77 \pm 20$ & 49 & $43 \pm 16$ & 30 & $380 \pm 180$ & 43 \\
apo AI KO & $19 \pm 3$ & 5 & $12 \pm 2$ & 5 & & $1.8 \pm 0.6$ \\
\end{tabular}

* Human apo AI. Tot. chol., total cholesterol; HDL-C, HDL cholesterol.

rabbit polyclonal antibody to human apo AI ( $2 \mathrm{~h}$ at room temperature) in $2 \%$ milk in $10 \mathrm{mM}$ phosphate buffer. apo AI HDL species were visualized with ${ }^{125} \mathrm{I}$-streptavidin (Amersham, Arlington Heights, IL). Unbound ${ }^{125} \mathrm{I}$ was washed four times with $1 \%$ milk in phosphate buffer, and nitrocellulose membranes were exposed to Fuji RX film at $-70^{\circ} \mathrm{C}$. No cross-reactivity was observed between this antibody and the murine apo AI.

Statistical analysis. Quantitative variables were expressed as mean \pm SD. Each $n$ represents the serum from an individual mouse. Linear correlation coefficients were used to describe relations between fractional efflux, esterification, and serum lipid and lipoprotein values. Regression lines for different data sets were compared by testing the identity of the slopes using the null hypothesis to calculate the $P$ value from a Fisher ratio (F test) (32), using DOS-based computers and a GraphPad (San Diego, CA) PRISM software program.

\section{Results}

Biochemical parameters of mouse serum. Sera from five groups of mice were used for studies of cholesterol efflux from Fu5AH cells: background, human apo AI transgenic (HuAITg), human CETP transgenic (HuCETPTg), human apo AI, and CETP transgenic (HuAICETPTg) and mouse apo AI knockouts (AI $\mathrm{KO}$ ). The lipoprotein changes in the different transgenic mice have been described previously in detail $(14,18,19,21)$. Table I shows that, as expected, all of the genetically engineered mice exhibited significant differences in total and HDL cholesterol concentrations compared with the background mice. In the $\mathrm{Hu}-$ AITg mice, HDL cholesterol was approximately doubled and represented $80 \%$ of the total cholesterol. On the other hand, as previously demonstrated (14), in both the HuCETPTg and HuAICETPTg mice, the HDL cholesterol was reduced markedly when compared with the background and HuAITg mice. Moreover, both groups expressing CETP had a similar HDL cholesterol concentration, and this represented $\sim 40 \%$ of total cholesterol, a much lower value than observed with the background animals. Human apo AI was present at very high concentrations in mice expressing the human apo AI gene $(580 \pm 200 \mathrm{mg} / \mathrm{dl})$, whereas the concentration was lower in mice having both human apo AI and human CETP (380 \pm 180 $\mathrm{mg} / \mathrm{dl}$ ). As anticipated, a significant correlation was observed between the serum total HDL cholesterol and human apo AI concentrations in HuAICETPTg mice $(r=0.73 ; P<0.001, n$ $=28)$ and HuAITg mice $(r=0.35 ; P<0.05, n=40)$. The expression of CETP was similar in both groups of mice expressing human CETP (Table I), and these concentrations were comparable with those usually reported in human serum (4). Both total and HDL cholesterol were reduced by $85 \%$ in $\mathrm{AI}$ KO mice (Table I).
Efflux to serum from transgenic mice. Dose-response studies determining the fractional efflux of labeled cholesterol from Fu5AH hepatoma cells incubated for $4 \mathrm{~h}$ with increasing concentrations of a pool of background mouse serum indicated that mouse serum was somewhat more efficient in stimulating efflux than human serum. A dilution of $5 \%$ serum, similar to that used in human serum studies (13), was selected for subsequent assays. Fig. 1 is a scattergram of all of the 4-h cholesterol efflux data, expressed as percent efflux, obtained with samples of serum from individual mice from each group. There was no difference in the percent efflux obtained with background and $\mathrm{Hu}$ AITg sera $(25.4 \pm 4.1$ vs. $26.3 \pm 3.3 \%)$. In contrast, we observed a progressive and significant decrease in the mean fractional efflux values in the HuCETPTg group $(20.7 \pm 3.2 \%)$, the $\mathrm{Hu}-$ AICETPTg group $(17.6 \pm 3.3 \%)$, and the $\mathrm{AI} \mathrm{KO}$ group $(6.5 \pm 0.5 \%)$, and these decreases in efflux paralleled decreases in serum HDL concentrations. The comparison of average efflux values among the transgenic animals shows that the fractional efflux was significantly lower in the HuAICETPTg than in either the HuAITg $(P<0.001)$ or the HuCETPTg $(P<0.05)$ groups. In addition there was a significant difference in efflux between the HuAITg and HuCETPTg mice $(P<0.001)$. Both male and female animals were used in these studies, based on availability. A comparison of the percent efflux values obtained with sera from male and female mice indicated that, although there may have been a trend for male animals to have somewhat greater efflux, there was no statistically significant difference

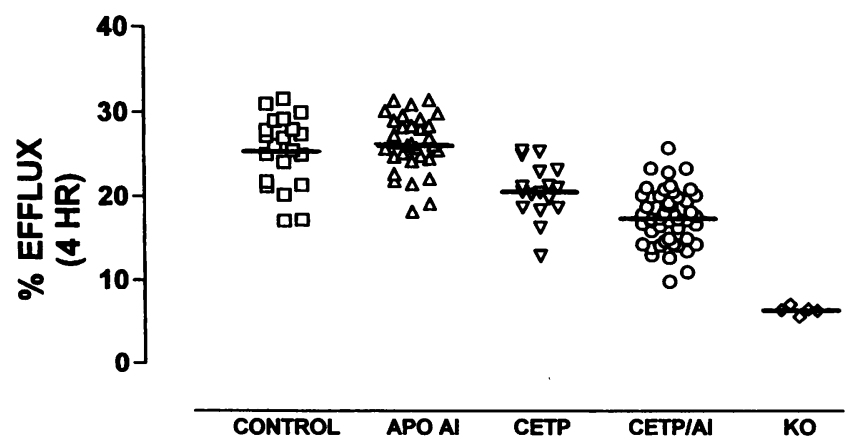

Figure 1. Scattergram of the percent efflux of cholesterol from Fu5AH cells exposed to serum (5\%) from genetically engineered mice (APO $A I$, HuAITg; CETP, HuCETPTg; CETP/AI, HuAICETPTg; $K O$, apo AI knockouts). Data are calculated for the efflux obtained after $4 \mathrm{~h}$ of exposure to the sera at $37^{\circ} \mathrm{C}$. Each data point represent an individual animal and the value is the average of triplicate determinations. The line illustrates the average percent efflux for each group of animals. 
Table II. Corelation between Cholesterol Efflux and HDL Cholesterol $(H D L-C)$ in Serum from Transgenic Mice and Analysis of the Slopes of the Regression Lines

\begin{tabular}{|c|c|c|c|c|c|c|c|}
\hline \multirow[b]{2}{*}{ Mice } & \multirow[b]{2}{*}{$n$} & \multirow{2}{*}{$\begin{array}{l}\text { HDL-C vs. } \\
\text { percent } \\
\text { efflux }\end{array}$} & \multirow{2}{*}{$\begin{array}{c}\text { Slope } \\
\text { (REP**) }\end{array}$} & \multicolumn{2}{|c|}{ Comparison vs. control } & \multicolumn{2}{|c|}{$\begin{array}{l}\text { Comparison vs } \\
\text { HuAICETPTg }\end{array}$} \\
\hline & & & & F & $P$ & $\mathbf{F}$ & $P$ \\
\hline & & $r^{2}$ & & & & & \\
\hline Background & 14 & $0.76 *$ & 0.24 & & & & \\
\hline HuAITg & 16 & $0.65^{*}$ & 0.09 & 12.90 & 0.001 & 7.47 & 0.01 \\
\hline HuCETPTg & 10 & $0.64 *$ & 0.32 & 0.96 & NS & 6.92 & 0.01 \\
\hline HuAICETPTg & 30 & $0.75 *$ & 0.16 & 4.84 & 0.03 & & \\
\hline
\end{tabular}

Linear correlation coefficients $\left(r^{2}\right)$ describing the relations between efflux of cell cholesterol from Fu5AH cells to medium containing the various mouse sera diluted to $5 \%$. Efflux was measured using standard assay conditions $\left(4 \mathrm{~h}, 37^{\circ} \mathrm{C}\right)$. The identity of the slopes was compared using an $\mathrm{F}$ test $(F)$. * Statistical significance was considered for $P<0.05$. ** REP, relative efflux potential.

based on gender in any group. In addition, the trend toward higher efflux with male serum was paralleled by a trend for higher HDL levels in the male mouse serum.

The fractional efflux values presented in Fig. 1 were obtained after a 4-h incubation period. This time was selected because it provided sufficient radioactivity to easily determine the amount of labeled cholesterol released and the fraction of this cholesterol that was esterified by serum LCAT (percent esterification). However, in a number of instances the percentage of cell cholesterol released to the medium was also determined after $30 \mathrm{~min}$ of incubation. There was a very high correlation between 30-min and 4-h efflux values $\left(r^{2}=0.85, n=81\right)$ when all groups were pooled. Thus, the similarities and differences we observed in the efflux potential of the sera from different groups of transgenic mice are not reflections of the length of incubation, and are the same for both relatively long $(4 \mathrm{~h})$ and relatively short (30 $\mathrm{min})$ incubations.

The number of samples in each group was sufficient (with the exception of the AI KO mice) to allow us to establish correlations between fractional efflux (\% efflux/4 $\mathrm{h}$ ) and the measured serum parameters. Table II shows that in all groups of animals the cholesterol efflux values were strongly, and positively, correlated with HDL cholesterol concentrations, which appeared to account for from 64 to $76 \%$ of the variation in the efflux. In contrast, human apo AI concentrations were not related to efflux in the HuAITg group and only weakly correlated in the HuAICETPTg group $\left(r^{2}=0.35, P<0.02\right)$. The CETP concentrations were negatively correlated to fractional efflux only in the HuAICETPTg group $\left(r^{2}=0.45, P<0.02\right)$.

A paradoxical observation was the lack of an increased fractional efflux in the HuAITg animals compared with background mice (Fig. 1), even though there was a large increase in HDL cholesterol (Table I) and a very strong correlation between fractional efflux and HDL cholesterol concentrations in the HuAITg group (Table II). The reason for this anomaly became apparent upon examination of the slopes of the regression lines fitting efflux to HDL cholesterol concentrations for each group of mice (Table II) which reflect the relative efficiency of the HDL in promoting cholesterol efflux (13). We term this slope the relative efflux potential (REP). The REP was significantly lower in the HuAITg group than in all other groups of mice (Table II). This difference in slopes indicates that, for the same relative variation in HDL cholesterol, the change in percent efflux is less in the HuAITg group than in all other groups. On the other hand, the coexpression of human CETP together with human apo AI increased the REP above that observed with apo AI alone. We can conclude from our data that: $(a)$ HDL particles from HuAITg mice are less efficient as cholesterol acceptors than HDL from the background mice; $(b)$ despite a lower average efflux due to lower HDL cholesterol concentrations, HDL particles are more efficient in the HuCETPTg mice than in the background mice; and $(c)$ the coexpression of both human apo AI and CETP improves the efficiency of HDL particles in the HuAICETPTg mice when compared with the HuAITg mice.

To establish that the data derived from studies using the hepatoma cells as cholesterol donors were representative of other cell types, we measured the fractional efflux of cholesterol from cholesterol-enriched mouse L cell fibroblasts. In particular, we wished to confirm the lack of stimulation of efflux by $\mathrm{Hu}$ AITg serum compared with background serum. L cells were selected since we have demonstrated that when they are cholesterol enriched they undergo significant cholesterol efflux upon exposure to lipid-free or lipid-poor human apo AI (33), and we hypothesized that serum from HuAITg mice expressing high levels of apo AI contains some lipid-poor apo AI (see below). The average value for the fractional efflux to $5 \%$ serum at $4 \mathrm{~h}$ was $29.7 \pm 4.2 \%(n=4)$ for background mouse serum and $31.5 \pm 1.6 \%(n=4)$ for HuAITg mouse serum. Thus, even in a cell system in which cholesterol efflux is particularly sensitive to the presence of free apo AI, the efflux potential of serum from HuAITg mice was not different than serum from the background animals.

The data collected in the experiments presented above were obtained with cells exposed to the various mouse sera diluted to 5\%. Although the efflux dose-response curves are not linear, we have demonstrated previously $(25,34)$ that estimates of the maximum rate of cell cholesterol release $\left(V_{\max }\right)$ can be determined by exposing the cells to increasing concentrations of acceptor and analyzing the dose-dependent percent efflux data by the method of Hofstee (26). Such an analysis yields estimates of both $V_{\max }$ and the concentration of acceptor that results in $50 \%$ of the maximum rate of cholesterol release $\left(\mathrm{EC}_{50}\right)$. However, we have now determined that maximum efflux values are not intrinsic properties of the particular cell type, but can be influenced by the nature of the cholesterol acceptor $(34,35)$. $V_{\max }$ and $\mathrm{EC}_{50}$ values for each type of serum are presented in Table III. Maximum rates of cell cholesterol release ranged 
Table III. Estimation of $V_{\max }$ and $E C_{50}$ Values for Cholesterol Efflux from Fu5AH Cells Incubated with Mouse Sera

\begin{tabular}{lcc}
\hline \multicolumn{1}{c}{ Mice } & \multicolumn{1}{c}{$V_{\max }$} & EC $_{\text {so }}$ \\
\hline & $\% / h$ & $\%$ serum \\
Background & $17.5 \pm 1.5$ & $8.6 \pm 1.5$ \\
HuAITg & $13.1 \pm 0.6^{* \ddagger}$ & $7.5 \pm 0.7^{\ddagger}$ \\
HuCETPTg & $19.3 \pm 0.9^{*}$ & $15.8 \pm 1.2^{*}$ \\
HuAICETPTg & $15.4 \pm 1.4^{\ddagger}$ & $11.9 \pm 2.2^{* \ddagger}$
\end{tabular}

Fu5AH cells incubated with sera $(2.5-30 \%)$ for $30 \mathrm{~min}$. Each group represents a pool of serum obtained from five mice. HDL cholesterol values $(\mathrm{mg} / \mathrm{dl})$ for the pools were: background $=80$; HuAITg $=113$; HuCETPTg $=59 ;$ HuAICETPTg $=73$. ${ }^{*}$ Significantly different $(P$ $<0.05$ ) from background; " significantly different from HuCETPTg. $V_{\max }$ and $\mathrm{EC}_{50}$ values were calculated as presented in Methods.

between 13 and $19 \%$ cell cholesterol/h, with $\mathrm{EC}_{50}$ values between 7.5 and $15.8 \%$ serum.

Esterification of the released cellular cholesterol. The quantification of the fractional esterification of the cholesterol released from the cells was accomplished by determining the distribution of the labeled cholesterol between the free and ester bands separated by TLC (Fig. 2). The average value for the fractional esterification of cell-derived cholesterol at the end of $4 \mathrm{~h}$ of incubation was $23.7 \pm 6.6 \% / 4 \mathrm{~h}$ in the background mouse serum. This value is much higher than the average value obtained with human serum $(6 \pm 1 \% / 4 \mathrm{~h})(13)$ under the same conditions of dilution and incubation. In the HuAITg group the fractional esterification was significantly lower than in the background mouse group $(13.6 \pm 3.1 \% / 4 \mathrm{~h} ; P<0.001)$. In contrast, the average esterification rate in both the HuCETPTg and HuAICETPTg groups, $24.3 \pm 6.0$ and $25.0 \pm 7.9 \% / 4 \mathrm{~h}$, respectively, was not different from the background group. Esterification in the apo AI knockout animals was $4.6 \pm 1.1 \% / 4 \mathrm{~h}$, which was significantly lower than that of any other group of animals. There were no significant correlations between the fractional efflux of cell cholesterol and the esterification of the released cholesterol, nor were there any significant correlations
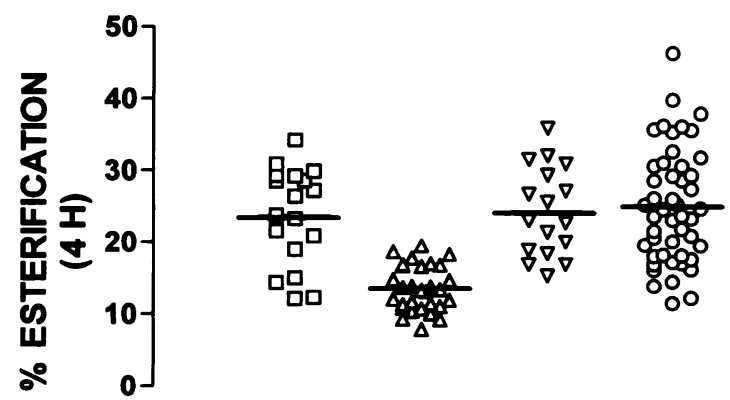

BKG

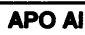

CETP

CETPIA

Figure 2. Scattergram of the percent esterification of the radiolabeled cholesterol that was released from Fu5AH cells incubated with sera (5\%) from background and transgenic mice. The specimens are the same as presented in Fig. 1. The values are the averages of triplicate determinations of the percentage of the released cholesterol recovered as cholesteryl ester after $4 \mathrm{~h}$ of incubation with cells at $37^{\circ} \mathrm{C}$. The line illustrates the average percent esterification for each group of animals.

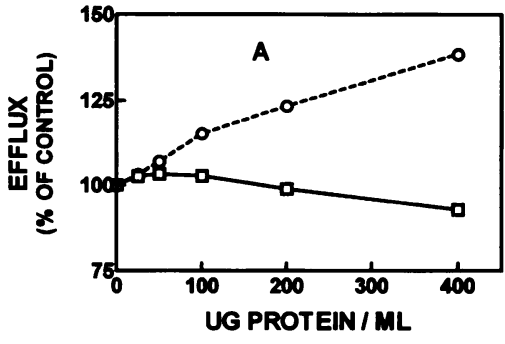

Figure 3. Modulation of cell cholesterol efflux and esterification of released cholesterol upon supplementation of background mouse serum with either human HDL or human lipid-free apo AI. HDL or apo AI were added to $100 \%$ serum at the indicated protein

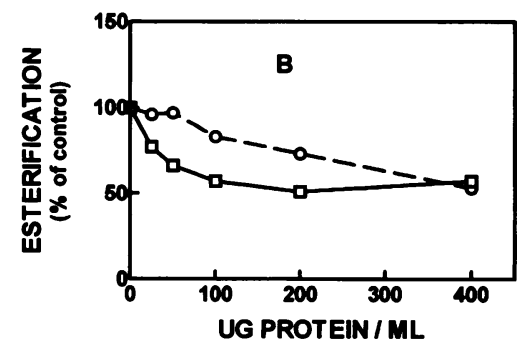
concentrations, after which the serum samples were diluted to $5 \%$ and incubated with Fu5AH cells labeled with $\left[{ }^{3} \mathrm{H}\right]$ cholesterol. Fractional efflux of labeled cholesterol and its esterification in the medium were measured after $4 \mathrm{~h}$ of in-

cubation. Each point represents the average of triplicate determinations. $\bigcirc$, human HDL; $\square$, lipid-free human apo AI. ( $A$ ) Cell cholesterol efflux; $(B)$, esterification of released cholesterol.

between esterification and serum parameters (i.e., total cholesterol, HDL cholesterol, human apo AI or human CETP) in any group.

Efflux to mouse serum supplemented in vitro with human apo AI or human HDL. To determine the extent to which cell cholesterol efflux could be modified by changing the concentrations in mouse serum of HDL or lipid-free apo AI, experiments were conducted in which Fu5AH cells were incubated with background mouse serum to which either human HDL or human apo AI was added in vitro. The range of HDL or human apo AI protein concentrations added to a background mouse serum pool was chosen to reproduce the range of apo AI concentrations obtained in the HuAITg animals. As shown in Fig. $3 A$, the effect of apo AI supplementation differed from that of HDL enrichment. In both cases, no stimulation of cholesterol efflux was observed with concentrations of apo AI $<1 \mathrm{mg} / \mathrm{ml}$. Increasing concentrations of apo AI as HDL particles stimulated, in a dose-dependent manner, the efflux induced by the mouse serum pool at a $5 \%$ concentration. In contrast, the addition of increasing amounts of lipid-free human apo AI had no stimulatory effect on the efflux induced by the mouse serum. Thus, in this experiment, the ability of human apo AI to promote increased cholesterol efflux to whole mouse serum is dependent on its presence as a lipid-containing particle. Fig. $3 B$ illustrates the effect of the addition of lipid-free human apo AI or HDL on the esterification of the cell-derived cholesterol. We observed a marked inhibition of esterification by all concentrations of lipid-free apo AI, whereas HDL particles progressively decreased the rate of esterification of released cholesterol in a dose-dependent manner.

Electrophoretic characterization of HDL species. The size of the HDL particles in each group of animals was assessed by nondenaturing GGE of serum prestained with Sudan black B (Fig. 4). As described previously $(14,18,21,36)$, HDL from HuAITg and HuAICETPTg mice exhibited a heterogeneous population of particles, whereas HuCETPTg HDL consisted of a single size population that is smaller than the HDL of the background mice. Blotting of the gels with anti-mouse apo AI 


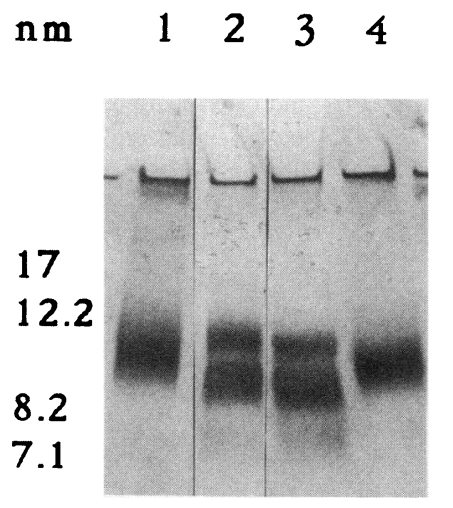

Figure 4. Distribution of HDL particle size in background and transgenic mice. Serum samples were stained with Sudan black before electrophoresis in native $4-15 \%$ polyacrylamide gradient gels. Lipid-stained lipoproteins were detected in background (lane 1), HuAITg (lane 2), HuAICETPTg (lane 3), and HuCETPTg (lane 4) mouse sera.

antibody indicated that, as expected, the levels of this apoprotein were reduced markedly in mice expressing human apo AI (data not shown $)(18,21)$.

The distribution of human apo AI among the HDL subspecies in HuAITg was examined by two-dimensional nondenaturing GGE (Fig. $5 \mathrm{~B}$ ). About $85 \%$ of the human apo AI was found in three $\alpha$-migrating HDL subspecies, corresponding to the human $\mathrm{HDL}_{2 \mathrm{a}}, \mathrm{HDL}_{2 \mathrm{~b}}$, and $\mathrm{HDL}_{3}$. The remaining human apo AI was found to migrate as pre $\beta 1-\mathrm{HDL}$, described previously in human plasma $(7,11)$ (Fig. $5 \mathrm{~B}$ ). Interestingly, compared with human plasma, HuAITg mouse serum did not have larger size pre $\beta$-migrating HDL (pre $\beta 2$ - and $\operatorname{pre} \beta 3$-HDL), and this gel pattern was the same for both fresh plasma and frozen serum (data not presented). In addition, when lipid-free human apo AI was electrophoresed in the same two-dimensional
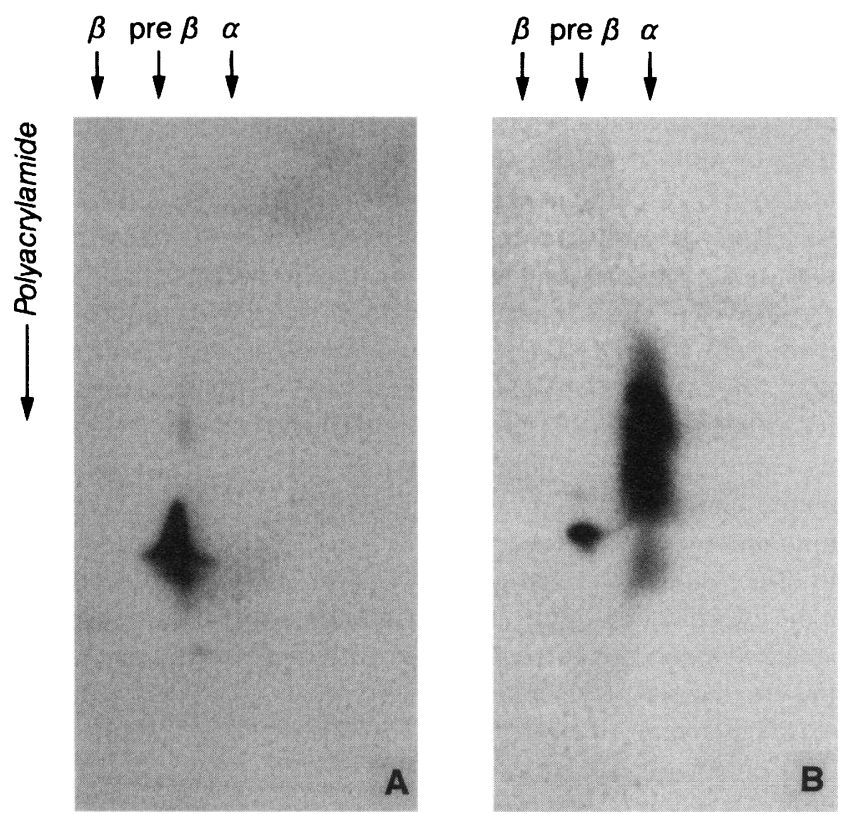

Figure 5. Distribution of lipid-free apo $\mathrm{AI}(A)$ and human apo $\mathrm{AI}(B)$ in HuAITg mice determined by nondenaturing two-dimensional gel electrophoresis. $15 \mu \mathrm{l}$ of human apo AI $(1 \mathrm{mg} / \mathrm{ml})$ or HuAITg mouse serum was run in agarose gel (first dimension) and a $3-16 \%$ polyacrylamide gel (second dimension). Proteins were transferred to nitrocellulose membranes and the human apo AI was visualized with rabbit antihuman apo AI antibody as described in Methods.
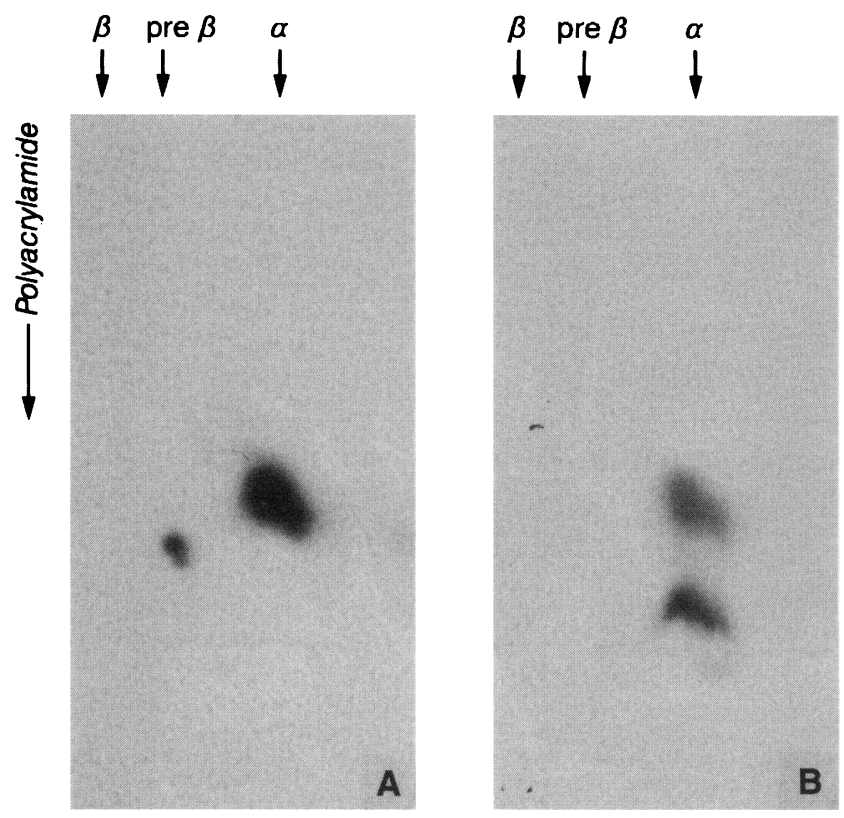

Figure 6. Distribution of human apo $\mathrm{AI}(A)$ and human $\mathrm{HDL}_{3}(B)$ in mouse serum. Background mouse serum was supplemented with 400 $\mu \mathrm{g} / \mathrm{ml}$ of either purified human apo $\mathrm{AI}$ or $\mathrm{HDL}_{3}$ and incubated for $4 \mathrm{~h}$ at $37^{\circ} \mathrm{C} .15 \mu \mathrm{l}$ of the supplemented serum was electrophoresed in a two dimensional electrophoresis system as described. The distribution of human apo AI was determined with a rabbit polyclonal antibody to human apo AI.

system, it exhibited a pattern corresponding to pre $\beta 1$-HDL (Fig. $5 A$ ).

The distribution of lipid-free human apo $\mathrm{AI}$ or human $\mathrm{HDL}_{3}$ added to background mouse serum was investigated using twodimensional, nondenaturing GGE. As shown in Fig. $6 \mathrm{~A}$, when purified lipid-free human apo AI was added to background mouse serum, the majority of the apo AI associated with a population of HDL particles in the $\alpha$-migrating region. Approximately $10 \%$ remained as lipid-free apo $\mathrm{AI}$ and migrated in the pre $\beta$-HDL region (Fig. $6 A$ ). In contrast, when human $\mathrm{HDL}_{3}$ was added to background mouse serum, human apo AI was associated only with $\alpha$-migrating particles and the size of these particles was similar to large $\mathrm{HDL}_{2 \mathrm{a}}$ and small $\mathrm{HDL}_{3}$ (Fig. $6 B)$.

\section{Discussion}

The ability of whole serum to promote the efflux of cholesterol from cells in culture has been quantified previously in a large number of human serum specimens and related to the apoprotein and lipoprotein components in the sera (13). Using whole serum as the acceptor of cell cholesterol provides the advantage that all of the serum components that contribute to the efflux of cholesterol and its subsequent metabolic steps are present. The transgenic mouse serum models that have been used in the present investigation provide the opportunity to study the selective effect of human lipoprotein-related proteins, namely apo AI and CETP, on efflux. The general system that was developed for the measurement of efflux potential of human serum (13) was easily applied to similar investigations using mouse serum, and because the experimental system used the 
serum after dilution to $5 \%$ we could assay a large number of samples from individual mice even though the quantity of serum obtained from each mouse is limited. With this system we determined the efflux potential of samples of serum obtained from background mice, mice expressing human apo AI, human CETP, or both apo AI and CETP. In addition, we were able to compare these results with values obtained with serum obtained from mice in which the expression of mouse apo AI had been inhibited.

The fractional efflux values for cholesterol released from the Fu5AH donor cells to the sera from the different groups of mice were in the order: background $=$ apo AI transgenic $>$ CETP transgenic $>$ apo AI/CETP transgenic $\gg$ apo AI KO (Fig. 1). The markedly reduced efflux to serum lacking the mouse apo AI demonstrates that apo AI-containing particles are primarily responsible for serum-stimulated efflux of cell cholesterol, consistent with studies demonstrating reduced efflux when apo AI-containing lipoproteins are removed by immunoaffinity chromatography (37). The absence of apo AI does not completely eliminate the efflux potential of serum, and from our results it can be estimated that $\sim 25 \%$ of the efflux potential of mouse serum is stimulated by lipoproteins lacking apo AI. The nature of these lipoproteins has not been established, but probably comprise apo AIV- $(38,39)$ and apo E- (40) containing particles.

The low average fractional efflux observed in both $\mathrm{Hu}-$ CETPTg and HuAICETPTg mice demonstrated that CETP expression produced a reduction in the ability of serum to remove cholesterol from cells which can be related to the lower HDL cholesterol concentrations in the serum. In contrast, the similarity in the average fractional efflux value of serum from background and HuAITg mice is unexpected since the overexpression of human apo $\mathrm{AI}$ in the transgenic animals results in elevated HDL cholesterol concentrations. Analysis of correlations between fractional efflux and serum components helps to clarify the differences in efflux potential observed between the different groups of transgenic mice. In every group, efflux best correlated to the concentrations of HDL cholesterol in the test sera. The high correlation between efflux and HDL cholesterol is consistent with our previous studies with human serum, except that the correlation coefficients obtained with all groups of mice are higher than obtained with human serum. This very good relationship between HDL and efflux may be a reflection of the predominance of HDL in mouse serum, and the data demonstrate that up to $80 \%$ of the variation in the efflux observed between individuals samples of mouse serum can be simply a reflection of the HDL concentration. Thus, in each group of mice there is a good correlation between efflux and HDL cholesterol, but the differences in HDL cholesterol between groups are not always reflected by proportional changes in fractional efflux. One possible explanation as to why serum from two groups of mice can have similar efflux potential, while having different HDL levels, is obtained from an examination of the slopes of the regression lines fitting efflux to HDL cholesterol for each group of mice (REP). Table II shows that the REP is 0.24 for the background animals and 0.09 for the apo AI transgene. We have previously interpreted the slopes of such lines as an indicator of the relative efficiency of a population of HDL particles to function as cholesterol acceptors, with an increased REP reflecting a greater ability of HDL to promote cholesterol efflux (13). Thus, it follows that the HDL particles present in the HuAITg animals are less efficient stimulators of cell cholesterol efflux than HDL in the background mice and the similarity in the average efflux value for background and HuAITg mice is a reflection of an increased amount of less efficient particles in the transgenic mice. It can be anticipated that if the concentrations of HDL were elevated to a greater extent in HuAITg animals, or if the HDL concentrations were reduced in normal mice, the expression of human apo AI could result in an apparent stimulation of cholesterol efflux. Thus, our results indicate that a determination of the efficiency of the serum in stimulating cholesterol efflux is more informative than a direct comparison of fractional efflux values or HDL cholesterol concentrations.

At this time it is impossible to definitively establish why the combination of HDL particles present in HuAITg serum are less efficient acceptors of cholesterol than HDL in the background animals. Based on a number of previous studies we propose that two parameters of HDL particles that play a critical role in modulating cell cholesterol efflux are particle size (41), and the amount and types of phospholipids associated with the various HDL particles $(34,42,43)$. In these initial studies we have not attempted to analyze the phospholipid composition of the various HDL particles in the serum; however, we have characterized the HDL in HuAITg serum as to relative size and charge. Agarose gel analysis of HuAITg serum has demonstrated previously that $\sim 20 \%$ of the human apo AI migrates as a pre $\beta$ species $(44,45)$, a value that we have confirmed in this study (data not presented). However, based on size and electrophoretic mobility, the nature of this pre $\beta$-migrating material cannot be definitively established since we have now demonstrated (Fig. 6) that lipid-free human apo AI cannot be distinguished from pre $\beta$-HDL (7) using the two-dimensional gel electrophoretic system routinely used for the detection of the small, pre $\beta$-HDL particles that have been postulated as the initial acceptors of cell cholesterol $(11,45)$.

In this study we are using the determination of HDL cholesterol as a quantitative measure of HDL particle concentration, which we have shown previously to correlate to the efflux potential of whole serum (13). However, we propose that HDL phospholipid would serve as a better predictor of efflux potential. At this time there are no quantitatively reliable data on the amount and types of phospholipids in the different HDL particles present in the serum of transgenic mice expressing either human apo AI or CETP. Because of the larger amounts of sera available from rats, we have obtained quantitative data on HDL phospholipid in rats expressing human apo AI, and these determinations have demonstrated that there is a disproportionate increase in apo AI, relative to phospholipid, in both the whole serum (46) and the HDL fraction (Fournier, N., and V. Atger, manuscript in preparation) of the apo AI transgenic rats, when compared with normal rats. The importance of HDL phospholipid is further exemplified by the experiment in which normal mouse serum was supplemented with either human lipid-free apo $\mathrm{AI}$ or $\mathrm{HDL}_{3}$. When apo $\mathrm{AI}$ is added to serum there is no change in phospholipid levels and no stimulation of efflux, even though the added apo AI could be detected in particles having the characteristics of $\mathrm{HDL}_{2}$ and pre $\beta$-HDL (Fig. 6). On the other hand, addition of $\mathrm{HDL}_{3}$ to the background mouse serum substantially stimulated efflux (Fig. 4). Thus, an overproduction of apo AI in apo AI transgenic animals, without a concomitant increase in available phospholipid, could result in the formation of lipid-free, or poorly lipidated, apo AI that would have pre $\beta$-mobility, but low capacity for cholesterol efflux. Studies 
are currently underway to characterize the HDL phospholipids in HDL from transgenic mice and to establish the changes that occur upon addition of either apo AI or phospholipids to serum from various sources.

The observation that the presence in serum of lipid-free or lipid-poor apo AI does not enhance efflux was unexpected in view of a number of publications demonstrating the ability of lipid-free apo AI (47-49) or lipid-free peptides (33) to promote the efflux of both cellular cholesterol and phospholipid. The extent of cholesterol efflux to lipid-free apoproteins varies considerably between cell types (50) and is enhanced by cell cholesterol enrichment $(33,49)$. However, in this study we observed no stimulation of efflux when cholesterol-enriched L cells served as cholesterol donors, even though these cells readily release cholesterol to lipid-free apo AI (33)and synthetic peptides (33). However, studies using lipid-free apoproteins as cell cholesterol acceptors have been conducted by adding the apoproteins to medium in the absence of lipoproteins and it has not been established to what extent lipid-free apoproteins will provoke greater efflux than that stimulated by the array of normal lipoproteins. The increased cell efflux obtained when background mouse serum was enriched with human HDL indicated that the lack of a stimulated efflux response with both HuAITg serum and lipid-free apo AI addition does not reflect a limitation of the cell system used in this study to release cholesterol, but rather a failure of the acceptors to stimulate efflux.

In this study we have observed that a major effect on efflux of the expression of human CETP in an animal that normally does not have this transfer factor is to lower the efflux potential of the serum. This effect is linked, at least in part, to the reduction in HDL cholesterol concentration. However, an analysis of the slopes of the regression lines (Table II) indicates a secondary effect of CETP in improving the relative efficiency of the HDL particles in removing cholesterol from cells. Indeed, the slope of the regression line between efflux and HDL cholesterol is higher in the HuCETPTg animals than in the background group. An even more convincing difference in slopes is obtained comparing the HuAITg with the HuAICETPTg mice. Based on these data it appears that the HDL particles in the serum of mice expressing both human CETP and apo AI are considerably more efficient acceptors of cell cholesterol than the HDL in animals expressing only the human apo AI.

The estimation of $V_{\max }$ values indicated a somewhat lower $V_{\max }$ for HuAITg serum when compared with background serum, and a reduced $V_{\max }$ for HuAICETPTg compared with HuCETPTg. Thus it appears that the expression of human apo AI reduces the estimated maximum rate of cell cholesterol release (Table III). Although a strict interpretation of the aqueous diffusion model for cholesterol efflux would predict that the $V_{\max }$ for cholesterol efflux from a particular cell type should not be influenced by the nature of the acceptor, our recent studies have indicated that acceptor size and composition can have rather dramatic effects on the $V_{\max }$ value $(33-35,41)$. The nature of this phenomenon is currently being examined and although the difference in $V_{\max }$ seen with the mouse sera may reflect differences in acceptor properties, a more detailed explanation must await future studies using well-characterized acceptor particles. It can be estimated that the efflux that would be obtained at $100 \%$ serum ranges from 83 to $90 \%$ of the estimated $V_{\max }$ values. Perhaps of greater interest are the estimates of $\mathrm{EC}_{50}$ which range from 7.5 to $15.8 \%$ (Table III). Thus, acceptor lipoproteins at
$7-15 \%$ of the concentration in plasma would produce $50 \%$ of the maximum cell cholesterol efflux and this range of concentrations is similar to estimates of the concentration of lipoproteins in interstitial fluids $(51-53)$. Thus, if the $\mathrm{EC}_{50}$ values for peripheral cells exposed to interstitial fluids were similar to that of the Fu5AH cells used in this study, the cholesterol acceptor lipoprotein concentration of interstitial fluid would be at a sensitive level, since small changes in lipoprotein concentration would produce relatively large changes in efflux.

The assay used in this investigation allows for the measurement of the fractional esterification of the radiolabeled cholesterol released from the cells. As shown in Fig. 2, this esterification value is significantly reduced in serum from the HuAITg mice when compared with all other groups. Interestingly, the coexpression of CETP together with human apo AI restores the esterification values to background levels. The restoration of esterification by CETP could be linked to either the remodeling of the HDL particles so that they serve as better substrates for the LCAT or to a reduction in the level of lipid-free apo AI in the serum. Just the presence of human apo AI itself is not sufficient to reduce esterification, since the human apo AI in HuAICETPTg serum did not inhibit esterification. Further studies will be necessary to resolve the reasons for the differing esterification values.

The fact that the efflux potential of serum from mice expressing human apo AI and elevated HDL cholesterol levels is not substantially different from that of the normal background mice we used as controls raises a number of physiological questions. If RCT is the major mechanism underlying the protective effect of HDL against atherosclerosis, our results are in apparent disagreement with studies showing a reduction in atherosclerotic lesions in HuAITg mice $(15,36)$. However, in our study we have only measured the movement of cholesterol molecules from cells to serum. Although efflux is the first step in RCT, this pathway involves multiple subsequent reactions, and thus the capacity of serum to accept cell cholesterol would only be one of many factors influencing the balance between removal and deposition of cholesterol in the vessel wall. Clearly, many other factors such as the serum lipoprotein profile, as well as the natural propensity for lesion development of different strains of mice, need to be considered. In this regard our studies are not strictly comparable with studies of lesion formation since we have not measured the efflux potential of serum from mice fed high-cholesterol diets or sera from apo E KO (16) and apo E KO-expressing apo AI mice $(54,55)$ which appear to be excellent models for studies of atherogenesis. The observation that mice expressing human CETP show a significant decrease in lesion formation despite their reduced HDL levels (Hayek, T., X. C. Jiang, E. M. Rubin, A. Tall, and J. L. Breslow, manuscript in preparation) indicates the complexity of this process and points to the fact that the efficiency of specific lipoprotein particles in removing cell cholesterol may be as important as the concentration of these particles in serum or interstitial fluid.

The extrapolation of data from transgenic mouse systems to the metabolism of HDL in humans should be undertaken with caution; however, our results suggest a number of relevant conclusions. First, it is clear from the experiments with serum from apo AI KO mice that, although apo AI-containing lipoproteins are important mediators of cell cholesterol efflux, they are not the sole acceptor particles in serum. Second, it appears that a large overexpression of apo AI does not necessarily result in a proportional stimulation of efflux and actually can produce 
an inhibition of cholesterol esterification by LCAT. We suggest that two factors that play an important role in determining HDL acceptor efficiency are particle size and phospholipid content $(33,34,41)$, and that in the HuAITg mice the supply of phospholipid is not sufficient to permit the formation of HDL particles suitable for efficient cholesterol efflux. Finally, although the expression of CETP reduces efflux by reducing HDL levels, CETP appears to have the secondary effect of increasing the efficiency of one or more of the HDL populations. The implications of these observations should be considered when efforts are made to develop pharmacological approaches designed to increase apo AI production or inhibit CETP activity in humans.

\section{Acknowledgments}

We wish to thank Drs. M. C. Phillips, W. J. Johnson, and J. Breslow for their encouragement and advice. In addition, the authors thank V. Van Nguyen, G. Stoudt, R. W. Clark, L. Royer, and M. Haghpassand for their patience and excellent technical assistance.

This work was supported in part by grants HL-22633 (G. Rothblat), HL-21006 (A. Tall), HL-22682 (A. Tall), and a Minority Investigator Research Supplement (MIRS) grant from the National Heart, Lung and Blood Institute of the National Institutes of Health (M. de la Llera Moya); National Science Foundation research planning grant MCB9308279 (M. de la Llera Moya); North Atlantic Treaty Organization collaboration research grant 930317 (V. Atger); Allegheny-Singer Research Institute grant 95-045-3MCP (G. Rothblat); and support from Pfizer Inc.

\section{References}

1. Reichl, D., and N. E. Miller. 1989. Pathophysiology of reverse cholesterol transport. Insights from inherited disorders of lipoprotein metabolism. Arteriosclerosis. 9:785-797.

2. Glomset, J. A. 1968. The plasma lecithin:cholesterol acyltransferase reaction. J. Lipid Res. 9:155-167.

3. Brown, M. S., and J. L. Goldstein. 1983. Lipoprotein metabolism in the macrophage: implications for cholesterol deposition in atherosclerosis. Annu. Rev. Biochem. 52:223-261.

4. Tall, A. R. 1993. Plasma cholesteryl ester transfer protein. J. Lipid Res. 34:1255-1274

5. Fielding, C. J. 1991. Reverse cholesterol transport. Curr. Opin. Lipidol. 2:376-378.

6. Fielding, P. E., C. J. Fielding, R. J. Havel, J. P. Kane, and P. Tun. 1983 Cholesterol net transport, esterification, and transfer in human hyperlipidemic plasma. J. Clin. Invest. 71:449-460.

7. Francone, O. L., and C. J. Fielding. 1990. Initial steps in reverse cholesterol transport: the role of short-lived cholesterol acceptors. Eur. Heart J. 11:218-224.

8. Phillips, M. C., W. J. Johnson, and G. H. Rothblat. 1987. Mechanisms and consequences of cellular cholesterol exchange and transfer. Biochim. Biophys. Acta. 906:223-276.

9. DeLamatre, J., G. Wolfbauer, M. C. Phillips, and G. H. Rothblat. 1986. Role of apolipoproteins in cellular cholesterol efflux. Biochim. Biophys. Acta. 875:419-428.

10. Fielding, C. J., and P. E. Fielding. 1995. Molecular physiology of reverse cholesterol transport. J. Lipid Res. 36:211-228.

11. Castro, G. R., and C. J. Fielding. 1988. Early incorporation of cell-derived cholesterol into pre- $\beta$-migrating high-density lipoprotein. Biochemistry. 27:2529.

12. Roheim, P. S., L. Dory, M. Lefevre, and C. H. Sloop. 1990. Lipoproteins in interstitial fluid of dogs: implications for a role in reverse cholesterol transport. Eur. Heart J. 11:225-229.

13. de la Llera Moya, M., V. Atger, J. L. Paul, N. Fournier, N. Moatti, P. Giral, K. E. Friday, and G. H. Rothblat. 1994. A cell culture system for screening human serum for ability to promote cellular cholesterol efflux: relationships between serum components and efflux, esterification and transfer. Arterioscler. Thromb. 14:1056-1065.

14. Hayek, T., T. Chajek-Shaul, A. Walsh, L. B. Agellon, P. Moulin, A. R. Tall, and J. L. Breslow. 1992. An interaction between the human cholesteryl ester transfer protein (CETP) and apolipoprotein A-I genes in transgenic mice results in a profound CETP-mediated depression of high density lipoprotein cholesterol levels. J. Clin. Invest. 90:505-510.
15. Schultz, J. R., J. G. Verstuyft, E. L. Gong, A. V. Nichols, and E. M. Rubin. 1993. Protein composition determines the anti-atherogenic properties of HDL in transgenic mice. Nature (Lond.). 365:762-764.

16. Nakashima, Y., A. S. Plump, E. W. Raines, J. L. Breslow, and R. Ross. 1994. ApoE-deficient mice develop lesions of all phases of atherosclerosis throughout the arterial tree. Arterioscler. Thromb. 14:133-140.

17. Melchior, G. W., C. K. Castle, R. W. Murray, W. L. Blake, D. M. Dinh, and K. R. Marotti. 1994. Apolipoprotein A-I metabolism in cholesteryl ester transfer protein transgenic mice. Insights into the mechanisms responsible for low plasma high density lipoprotein levels. J. Biol. Chem. 269:8044-8051.

18. Rubin, E. M., B. Y. Ishida, S. M. Clift, and R. M. Krauss. 1991. Expression of human apolipoprotein A-I in transgenic mice results in reduced plasma levels of murine apolipoprotein A-I and the appearance of two new high density lipoprotein size subclasses. Proc. Natl. Acad. Sci. USA. 88:434-438.

19. Walsh, A., Y. Ito, and J. L. Breslow. 1989. High levels of human apolipoprotein A-I in transgenic mice result in increased plasma levels of small high density lipoprotein (HDL) particles comparable to human HDL3. J. Biol. Chem. 264:6488-6494.

20. Chajek-Shaul, T., T. Hayek, A. Walsh, and J. L. Breslow. 1991. Expression of the human apolipoprotein A-I gene in transgenic mice alters high density lipoprotein (HDL) particle size distribution and diminishes selective uptake of HDL cholesteryl esters. Proc. Natl. Acad. Sci. USA. 88:6731-6735.

21. Agellon, L. B., A. Walsh, T. Hayek, P. Moulin, X. C. Jiang, S. A Shelanski, J. L. Breslow, and A. R. Tall. 1991. Reduced high density lipoprotein cholesterol in human cholesteryl ester transfer protein transgenic mice. J. Biol. Chem. 266:10796-10801.

22. Plump, A. S., T. Hayek, A. Walsh, and J. L. Breslow. 1993. Diminished HDL cholesterol ester flux in apo A-I-deficient mice. Circulation. 88:I-422a. (Abstr.)

23. Gallo, L. L., R. Atasoy, G. V. Vahouny, and C. R. Treadwell. 1978 Enzymatic assay for cholesterol ester hydrolase activity. J. Lipid Res. 19:913916.

24. Clark, R. W., J. B. Moberly, and M. J. Bamberger. 1995. Low level quantification of cholesteryl ester transfer protein in plasma subfractions and cell culture media by monoclonal antibody-based immunoassay. J. Lipid Res. 36:890900

25. Davidson, W. S., S. Lund-Katz, W. J. Johnson, G. M. Anantharamaiah, N. Palgunachari, J. P. Sergrest, G. H. Rothblat, and M. C. Phillips. 1994. The influence of apolipoprotein structure on the efflux of cellular free cholesterol to high density lipoprotein. J. Biol. Chem. 269:22975-22982.

26. Segel, I. H. 1976. Biochemical Calculations. John Wiley \& Sons Inc., New York. 235 pp.

27. Arbogast, L. Y., G. H. Rothblat, M. H. Leslie, and R. A. Cooper. 1976. Cellular cholesterol ester accumulation induced by free cholesterol-rich lipid dispersions. Proc. Natl. Acad. Sci. USA. 73:3680-3684.

28. Bligh, E. G., and W. J. Dyer. 1959. A rapid method of total lipid extraction and purification. Can. J. Biochem. 37:911-917.

29. Atger, V., D. Malon, M. C. Bertiere, F. N'Diaye, and A. Girard-Globa. 1991. Cholesterol distribution between high-density-lipoprotein subfractions HDL2 and HDL 3 determined in serum by discontinuous gradient gel electrophoresis. Clin. Chem. 37:1149-1152.

30. Hussain, M. M., N. L. R. Bucher, B. Faris, C. Franzblau, and V. I. Zannis 1988. Tissue-specific posttranslational modification of rat apoE. Synthesis of sialated apoE forms by neonatal rat aortic smooth muscle cells. J. Lipid Res. 29:915923.

31. Francone, O. L., A. Gurakar, and C. Fielding. 1989. Distribution and functions of lecithin:cholesterol acyltransferase and cholesteryl ester transfer protein in plasma lipoproteins. J. Biol. Chem. 264:7066-7072.

32. Zar, J. H. 1984. Biostatistical Analysis. Prentice-Hall, New York. p. 62.

33. Yancey, P. G., J. K. Bielicki, W. J. Johnson, S. Lund-Katz, M. N. Palgunachari, G. M. Anantharamaiah, J. P. Segrest, M. C. Phillips, and G. H. Rothblat. 1995. The efflux of cellular cholesterol and phospholipid to lipid-free apolipoproteins and class A amphipathic peptides. Biochemistry. 34:7955-7965.

34. Davidson, W. S., K. L. Gillotte, S. Lund-Katz, W. J. Johnson, G. Rothblat, and M. C. Phillips. 1995. The effect of high density lipoprotein phospholipid acyl chain composition on the efflux of cellular free cholesterol. J. Biol. Chem. 270:5882-5890.

35. Kilsdonk, E. P. C., P. Yancey, G. Stoudt, F. W. Bangerter, W. J. Johnson, M. C. Phillips, and G. H. Rothblat. 1995. Cellular cholesterol efflux mediated by cyclodextrins. J. Biol. Chem. 270:17250-17256.

36. Rubin, E. M., R. M. Krauss, E. A. Spangler, J. G. Verstuyft, and S. M. Clift. 1991. Inhibition of early atherogenesis in transgenic mice by human apolipoprotein AI. Nature (Lond.). 353:265-267.

37. Fielding, C. J., and K. Moser. 1982. Evidence for the separation of albumin- and apo A-I-dependent mechanisms of cholesterol efflux from cultured fibroblasts into human plasma. J. Biol. Chem. 257:10955-10960.

38. Stein, O., Y. Stein, M. Lefevre, and P. S. Roheim. 1986. The role of apolipoprotein A-IV in reverse cholesterol transport studied with cultured cells and liposomes derived from an ether analog of phosphatidylcholine. Biochim. Biophys. Acta. 878:7-13. 
39. Steinmetz, A., R. Barbaras, N. Ghalim, V. Clavey, J.-C. Fruchart, and G. Ailhaud. 1990. Human apolipoprotein A-IV binds to apolipoprotein A-I/A-II receptor sites and promotes cholesterol efflux from adipose cells. J. Biol. Chem. 265:7859-7863.

40. Huang, Y., A. von Eckardstein, S. Wu, N. Maeda, and G. Assmann. 1994 A plasma lipoprotein containing only apolipoprotein $\mathrm{E}$ and with $\gamma$ mobility on electrophoresis releases cholesterol from cells. Proc. Natl. Acad. Sci. USA. 91:1834-1838.

41. Davidson, W. S., W. V. Rodrigueza, S. Lund-Katz, W. J. Johnson, G. H. Rothblat, and M. C. Phillips. 1995. Effects of acceptor particle size on the efflux of cellular free cholesterol. J. Biol. Chem. 270:17106-17113.

42. Burns, C. H., and G. H. Rothblat. 1969. Cholesterol excretion by tissue culture cells effect of serum lipids. Biochim. Biophys. Acta. 176:616-625.

43. Chau, I. Y., and R. P. Geyer. 1978. The effects of serum albumin and phospholipid on sterol excretion in tissue culture cells. Biochim. Biophys. Acta. 542:214-221.

44. Francone, O. L., E. L. Gong, D. S. Ng, C. J. Fielding, and E. M. Rubin. 1995. Expression of human lecithin-cholesterol acyltransferase in transgenic mice. Effect of human apolipoprotein AI and human apolipoprotein AII on plasma lipoprotein cholesterol metabolism. J. Clin. Invest. 96:1440-1448.

45. Asztalos, B. F., C. H. Sloop, L. Wong, and P. S. Roheim. 1993. Twodimensional electrophoresis of plasma lipoproteins: recognition of new apo A-Icontaining subpopulations. Biochim. Biophys. Acta. 1169:291-300.

46. Burkey, B. F., D. France, H. Wong, M. Xaiowen, B. Nottage, P. Diffenderfer, J. B. Marsh, J. R. Paterniti, and E. A. Fisher. 1995. Overexpression of human apolipoprotein $\mathrm{AI}$ ( $\mathrm{h}$-apo $\mathrm{AI}$ ) in transgenic rats suppresses the hyperlipoproteinemia associated with experimental nephrosis. J. Lipid Res. 36:1463-1473.
47. Hara, H., and S. Yokoyama. 1992. Role of apolipoproteins in cholesterol efflux from macrophages to lipid microemulsion: proposal of a putative model for the pre-B high-density lipoprotein pathway. Biochemistry. 31:2040-2046.

48. Hara, H., A. Komaba, and S. Yokoyama. 1992. Alpha-helical requirements for free apolipoproteins to generate HDL and to induce cellular lipid efflux. Lipids. 27:302-304.

49. Bielicki, J. K., W. J. Johnson, R. B. Weinberg, J. M. Glick, and G. H. Rothblat. 1992. Efflux of lipid from fibroblasts to apolipoproteins: dependence on elevated levels of cellular unesterified cholesterol. J. Lipid Res. 33:16991710 .

50. Komaba, A., Q. Li, H. Hara, and S. Yokoyama. 1992. Resistance of smooth muscle cells to assembly of high density lipoproteins with extracellular free apolipoproteins and to reduction of intracellularly accumulated cholesterol. J. Biol. Chem. 267:17560-17566.

51. Roheim, P. S. 1986. Atherosclerosis and lipoprotein metabolism: role of reverse cholesterol transport. Am. J. Cardiol. 57:3C-10C

52. Reichl, D. 1990. Lipoproteins of human peripheral lymph. Eur. Heart J. 11:230-236.

53. Reichl, D. 1994. Extravascular circulation of lipoproteins: their role in reverse transport of cholesterol. Atherosclerosis. 105:117-129.

54. Plump, A. S., C. J. Scott, and J. L. Breslow. 1994. Human apolipoprotein A-I gene expression increases high density lipoprotein and suppresses atherosclerosis in the apolipoprotein E-deficient mouse. Proc. Natl. Acad. Sci. USA. 91:9607-9611.

55. Pászty, C., N. Maeda, J. Verstuyft, and E. M. Rubin. 1994. Apolipoprotein AI transgene corrects apolipoprotein E deficiency-induced atherosclerosis in mice. J. Clin. Invest. 94:899-903. 